Journal de la Société des Océanistes

\section{Journal de la Société des Océanistes}

140 | janvier-juin 2015

Intégration régionale des territoires français dans le Pacifique Sud

\title{
Les transformations du politique : leaderships et question environnementale en Polynésie française
}

Politics in Change, Leaderships and the Environmental Issue in French Polynesia

\section{Rudy Bessard}

\section{OpenEdition}

\section{Journals}

Édition électronique

URL : http://journals.openedition.org/jso/7273

DOI : $10.4000 /$ jso.7273

ISSN : $1760-7256$

Éditeur

Société des océanistes

Édition imprimée

Date de publication : 15 juin 2015

Pagination : 89-104

ISBN : 9782854301250

ISSN : 0300-953x

Référence électronique

Rudy Bessard, «Les transformations du politique : leaderships et question environnementale en Polynésie française », Journal de la Société des Océanistes [En ligne], 140 I janvier-juin 2015, mis en ligne le 05 juillet 2015, consulté le 13 novembre 2019. URL : http://journals.openedition.org/jso/7273 ; DOI : 10.4000/jso.7273 


\title{
Les transformations du politique : leaderships et question environnementale en Polynésie française
}

par

\author{
Rudy BESSARD*
}

\section{RÉSUMÉ}

L'importance croissante des transformations environnementales (changement et réchauffement climatique, pollutions, énergies...) confronte le champ du politique à la réflexion et à l'action sur ces sujets. Les territoires français du Pacifique Sud sont impactés par ces mutations accélérées. A partir de l'exemple de la Polynésie française, cet article interroge les formes de leadership institutionnel et politique dans leurs rapports à la question environnementale. L'État et les principaux leaderships politiques polynésiens mobilisent ainsi des ressources politiques qui varient entre ressources institutionnelles, identitaires et rentières, parfois en décalage avec le renouvellement des enjeux politiques et la mise à l'agenda des problématiques écologiques.

MoTS-CLÉs : leadership, transformations, ressources politiques, environnement, État, Polynésie française, Pacifique

\begin{abstract}
The political field is more and more confronted to the increasing dimension of environmental transformations (climate change, pollutions, energy issues...). In the South Pacific, the French territories are impacted by these accelerated mutations. The article, which is based on the example of French Polynesia, deals with the institutional and political leadership relation to the ecological issue. Thus, the State and most Polynesian political leaderships are mobilizing political resources - institutional, identity and rent resources - that make appear the gap with renewed political stakes and the environmental agenda.
\end{abstract}

KEYwords: leadership, transformation, political resources, environment, Pacific, French Polynesia

biotechnologies, de sciences de l'information/ communication et des sciences cognitives, dans un processus accéléré à même de modifier les sociétés humaines (Poirson et Miailhe, 2013). Dans ce contexte, les défis politiques s'articulent de plus en plus en fonction d'enjeux mondialisés en termes de domination (Hibou, 2011), de démocratie (Milacic, 2006), de rivalités économiques (Stiglitz, 2008) et de ressources géopolitiques liées au contrôle des océans (Royer, 2012). Les transformations globales expriment l'apparition d'un nouveau monde encore peu déchiffrable (Morin, 2007), dont les nouvelles potentialités et contraintes n'écartent pas de leur spectre la "mer d'îles" océaniennes

* Politiste, chercheur associé au Centre Montesquieu de recherches politiques (CMRp, Université de Bordeaux) et au laboratoire Gouvernance et développement insulaire (GDI, Université de Polynésie française), rbessard7@gmail.com 
(Hau’Ofa, 2008). Ainsi, les collectivités françaises $\mathrm{du}$ Pacifique (Nouvelle-Calédonie, Polynésie française, Wallis-et-Futuna), qui présentent des spécificités contrastées (voir l'introduction du dossier), sont confrontées à ces mutations, en particulier sur le plan environnemental (Trichet et Leblic, 2008) ; la Nouvelle-Calédonie est ainsi un "point chaud" de la biodiversité mondiale confrontée à l'exploitation minière (Richer de Forges et Pascal, 2008). Au moment où la $21^{\mathrm{e}}$ conférence internationale Paris Climat 2015 se déroulera à la fin de l'année 2015 en France (http:// www.cop21.gouv.fr/fr) et à laquelle la présidence de François Hollande attache une importance particulière, la question environnementale résonne mondialement dans l'attente d'interventions politiques permettant un accord universel sur le climat. En Océanie, la Polynésie française fait partie des principaux territoires directement concernés par les dérèglements climatiques et la transition énergétique. À partir du cas polynésien, cet article interroge les différentes formes de leadership politique dans leur rapport avec les transformations environnementales. De quelle manière ces leaderships mobilisent-ils des ressources politiques ou institutionnelles pour répondre à l'émergence de nouveaux enjeux environnementaux, climatiques et énergétiques? Le texte s'appuie sur des éléments collectés sur le terrain de recherche (données scientifiques, littérature en sciences humaines, rapports institutionnels, articles de presse et entretiens), qui avaient été mis de côté durant la thèse, soutenue en 2013, puis, accumulés lors de séjours à Tahiti en février-mars et juin 2014 dans le cadre des activités de recherche en science politique ${ }^{1}$.

\section{Politique et environnement}

L'apparent paradoxe entre le rôle fondamental du politique et la nature des enjeux technologiques et environnementaux, tels que la "démocratie numérique » (Cardon, 2010 ; Casilli, 2010) ou la "démocratie écologique " (Bourg et Whiteside, 2010) par exemple, peut s'expliquer à première vue par les différentes temporalités des champs d'action qui leurs sont relatifs. À la lenteur du politique s'oppose la vitesse de transformations mondiales accélérées et interconnectées. L'étude séparée des transformations environnementales d'un côté et technologiques de l'autre n'a de sens que pour l'intelligibilité du propos. Ainsi, la notion de "techno-environnement " a été définie à partir de deux éléments qui font système :
"L'environnement utile d'une société donnée est donc relatif aux techniques et aux savoirs dont elle dispose. Réciproquement, les sociétés produisent les savoirs et les techniques appropriées à un environnement donné. » (Laburthe-Tolra et Warnier, 2003 : 330-331)

Dans cette optique, le champ de l'environnement étant désormais mondial, les sociétés humaines produisent des connaissances et des technologies à un rythme jamais observé dans l'histoire humaine. La réponse du politique face à ce constat oscille entre discours alarmistes, proclamations d'intérêt général et une action publique d'une ampleur insuffisante. Le renouvellement d'enjeux politiques universels entrecroisés implique pourtant la mise en relation des enjeux environnementaux avec les problématiques sociales et politiques. La démarche théorique d'Hannah Arendt définit la politique comme un espace relationnel - "La politique prend naissance dans l'espace intermédiaire et elle se constitue comme relation"-, qui "organise d'emblée des êtres absolument différents en considérant leur égalité relative et en faisant abstraction de leur diversité relative" (1995: 43). De fait, penser l'espace public ou commun conduit à mettre en évidence les enjeux relatifs aux questions environnementale - et technologique: par exemple, les projets de "maison amphibie » ou de "bateau-nation" (Le Monde, 3-4/02/2008) -, souvent délaissés ou peu approfondis dans le débat politique.

Par la conciliation d'apparentes contradictions et d'intérêts opposés, la politique peut aussi être analysée en tant que conflit dont le sujet a vocation à s'universaliser, ou, comme un affrontement constant entre deux processus de gouvernement ou de police - au sens d'activité ordonnatrice de la société - et d'égalité, au sens du jeu des pratiques relatives à l'émancipation (Rancière, 2004).

Les mutations environnementales, mais aussi l'attractivité des nouveaux médias ou des technologies dans ces territoires, montre la capacité d'accueil des innovations dans les sociétés du Pacifique, qui appelle à désenclaver la pensée au sujet de territoires qui ne sont pas prisonniers d'une altérité indépassable. D'ailleurs, un intérêt mutuel entre les îles du Pacifique et « le reste du monde " pourrait être décelé dans l'échange entre la mondialisation et les systèmes de pensée ou de valeurs véhiculés en Océanie (Babadzan, 2009). Certains de ces aspects portent également une vision non séparée des rapports entre l'homme et la nature, envisageant plutôt le cosmos comme un ensemble (Saura, 2008). C'est d'ailleurs sous cet angle que le sénateur polynésien Richard Tuheiava ${ }^{2}$ présente ses conceptions :

1. Je tiens à remercier les lecteurs et correcteurs de la première version de ce papier proposé au Journal de la Société des Océanistes pour la qualité de leurs remarques, ce qui a permis à cet article de prendre la forme finale présentée ici.

2. Indépendantiste modéré du parti Tavini Huiraatira, qui développe une approche ouverte du souverainisme par rapport à l'approche radicale du président du parti, Oscar Temaru. 
"Cette façon de vivre, cette croyance, cette cosmogonie mérite de participer et de contribuer à l'universel. » (Massau, $2010:$ 16)

Par ailleurs, articuler la question des transformations environnementales avec le politique appelle à la prise en compte des apports de l'anthropologue Bruno Latour, qui a posé sans détour le problème $\mathrm{du}$ dialogue entre les sciences studies et la science politique, qui s'appuie sur des données élaborées dans d'autres disciplines (Latour, 2002 ; 2008). Toutefois, plusieurs limites à ces perspectives ont été relevées par Pierre Favre, en particulier sur la l'absence de séparation entre "science" et "politique» (Favre, 2008). Sans se perdre dans les méandres du débat sur l'identification des objets politiques, il reste que les problèmes environnementaux appellent des réponses publiques, définies dans le cadre de politiques publiques, ou intégrées dans le cadre de stratégies des acteurs ou des partis politiques.

La manifestation sociale de différentes formes de leadership en rapport, par leurs discours ou leurs actions, avec la problématique environnementale, développent une portée politique, dès lors qu'on entend le politique par la non-réciprocité, autrement dit "ce qui ne se donne ni ne s'échange" (Seiler, 2006). Le caractère souverain du politique ne remet cependant pas en cause l'approche en termes de science politique de l'ensemble des entités politiques du Pacifique, bien qu'elles ne jouissent pas toutes des attributs de la souveraineté étatique.

Ainsi, plusieurs défis politiques dans le Pacifique insulaire concernent la définition des formes futures de l'architecture institutionnelle ou juridique de ces territoires, ainsi que l'évolution de systèmes politiques très divers dans la région. Ces écosystèmes politiques, autant de Pacific Ways (Levine, 2009) selon le politiste Stephen Levine, sont marqués par une histoire, et une culture spécifiques, ainsi que par un pluralisme juridique (Bambridge et Rigo, 2012). Ils sont néanmoins inscrits dans la mondialisation (Demmer et Salaün, 2006) et dans la modernité politique. Les individus votent, se présentent aux élections, forment des partis politiques, expriment leur attachement pour un leader, un gouvernement, l'opposition, etc. Les formes de la démocratie ne sont pas le calque des modèles tels qu'ils sont pratiqués dans les pays occidentaux, mais ils n'en demeurent pas moins dépourvus de "sens démocratique »(Wittersheim, 2006 ; 2014).

\section{Leadership et transformations environnementales}

Le caractère spécifique du politique est décelable dans différents processus de légitimation des leaderships politiques ou institutionnels en mesure de faire face à la complexité des mutations contemporaines. L'approche des leaderships politiques et sociaux est intéressante parce qu'elle s'avère interactive entre les transformations générales et les sociétés particulières. C'est-àdire que l'analyse des leaderships est heuristique, puisqu'elle permet de considérer les interactions entre les mutations globales et les sociétés politiques des territoires du Pacifique.

La notion de leadership, définie comme "la capacité d'un représentant à gérer de façon dynamique des rapports de pouvoir au sein d'un groupe" (Braud, 2004: 681), est mobilisée dans des travaux qui alimentent la dimension compréhensive des phénomènes de pouvoir chère à Max Weber. Ainsi, le leadership politique renvoie à des phénomènes de domination et de légitimité, d'échange et de réciprocité (Smith et Sorbets, 2003). Il se limite à ceux qui exercent leur autorité au nom de l'intérêt général et inclut les leaderships dont la légitimation est de nature institutionnelle ou sociale (États, organisations internationales, groupes sociaux, individus en situation depouvoir).Leleadershiptransactionnel, le plus courant en régime démocratique, se distingue du leadership de recomposition. Le leadership transactionnel se fonde sur "l'attente d'un échange mutuellement productif " (soutien par le vote contre promesses de réalisation). La dimension du clientélisme en tant qu'institution sociale apparaît dans cette approche. Elle est utile à la compréhension du contexte politique polynésien, dans lequel le clientélisme scande des rapports politiques personnalisés et représente le principal mode de médiation politique entre l'État et le territoire polynésien, entre autorités politiques nationales et notables tahitiens, ou entre l'électeur et son représentant. Le clientélisme peut aussi représenter une des formes de néopatrimonialisme. Ce concept, utile pour mettre en relief le chevauchement des ressources publiques et privées (Bessard, 2013: 569-654), englobe les pratiques de clientélisme, de corruption ou de népotisme. En outre, le recours à la ressource politique de la mobilisation identitaire appartient également aux manifestations du leadership transactionnel en Polynésie française, où les partis autonomistes et indépendantistes utilisent différemment la politisation du récit identitaire de la " polynésianité " (Celentano, 2002 ; Saura, 2008 ; Bessard, 2013 : 358-368).

Le leadership de recomposition correspond à l'attitude dynamique d'un dirigeant qui vise à transformer les cadres de la représentation. Ce leadership peut prendre une forme personnalisée ou idéologisée. Dans le cas d'un leadership personnalisé, l'interaction est basée sur «l'autorité charismatique" (Weber, 2004 [1921]) ou sur le "leadership hérö̈que" (Burns, 1978). $\mathrm{Si}$ le leadership politique classique exprime la recherche du maintien du statu quo (logique 
de conservation du pouvoir), le concept de leadership de la transformation, ou "leadership transformationnel ", représente un outil conceptuel dérivé de cette notion, qui provient de l'approche du leadership développée par James Burns dans le concept de transforming leadership (Burns, 2007). Elle a été notamment utilisée par Bernard Bass (transformational leadership) dans un registre managérial, en définissant quatre éléments d'un leadership transformationnel efficace: charisme, inspiration, stimulation intellectuelle et considération. L'organisation (ou l'institution) est revitalisée par l'énergie transmise par le leader. Dans sa forme normative, le leadership transformationnel modifie les valeurs et les attitudes des suiveurs pour atteindre des buts plus élevés (paix, justice, liberté), le plus souvent, en vertu d'une éthique. La fin de ce type de leadership arrive quand l'imposition des transformations devient impopulaire. Le passage d'un leadership transactionnel à un leadership transformationnel est marqué par la mutation d'un leader " courtier " à un leader acteur majeur d'un changement social en redistribuant du pouvoir à ses suiveurs à partir d'objectifs de long terme (Burns, 2007).

Appliqué à la sociologie politique, à la sociologie des organisations et à l'étude d'entrepreneurs mondialisés, le concept peut aussi être transposé de façon intéressante à l'échelle étatique, dans le sillage de la conception d'un État entrepreneur en matière d'innovation (Mazzucato, 2011). Cette approche schumpetérienne remet en cause certains dogmes opposés à l'interventionnisme étatique, en montrant le "court-termisme" du secteur privé. Pour autant, une critique similaire peut être formulée à propos de la professionnalisation de l'action politique, qui se détermine en fonction d'un temps électoral favorisant rarement une approche de long terme. Néanmoins, le recours à la catégorie de leadership transformationnel n'est pas toujours des plus éclairantes et nécessite donc une instillation parcimonieuse dans l'étude des leaderships.

L'identification des acteurs principaux vecteurs de leadership dans l'espace polynésien fait apparaître l'État, par l'action des services publics étatiques, la collectivité d'outre-mer de Polynésie française (assemblée, gouvernement et administration territoriale), ainsi que les principaux leaders et partis politiques. L' "étaticité " du dispositif politico-administratif polynésien révèle le caractère étatique d'institutions locales récentes et en cours de construction. Par ailleurs, les acteurs socio-économiques ne sont pas exclus du champ $\mathrm{du}$ leadership mais ne font pas directement partie de cette étude. Néanmoins, les entrepreneurs s'inscrivent souvent dans une logique d'entreprise " politico-économique »s'appuyant sur différentes formes de capitaux. En Nouvelle-Calédonie par exemple, les entrepreneurs politiques mobilisent une matrice des ressources en jouant à la fois sur les registres politique, économique, social, minier, foncier, etc. (Le Meur, 2014).

Enfin, la Polynésie française, associée en tant que Pays et Territoire d'Outre-Mer (Ртом) à l'Union européenne, fait de cette dernière un acteur complémentaire en matière de coopération institutionnelle régionale et de dotation de fonds de développement non négligeables mais peu abondants pour les ртом français (Budoc, 2012). Toutefois, le $11^{\mathrm{e}} \mathrm{FED}$ régional mobilise les thématiques du changement climatique et des énergies renouvelables, ainsi qu'il en a été acté lors de la conférence ministérielle annuelle de l'Association des ртом (оста) et du 13 $13^{\text {e }}$ Forum de dialogue politique UE-PTOM, réunis les 26 et 27 février 2015 aux îles Vierges britanniques. Le développement de la thématique environnementale dans les politiques européennes contribue ainsi à orienter les politiques publiques territoriales dans les secteurs vecteurs de fonds européens. Selon Gaston Tong-Sang, ancien président autonomiste entre 2006 et 2010, il s'agit aussi de rééquilibrer les relations entre l'Union européenne et les pays d'outre-mer, afin que ceuxci ne soient plus considérés comme des pays en voie de développement, pour devenir :

«[...] des têtes de pont de l'ue dans la région [...]. Nous devons pouvoir utiliser l'ue comme une caisse de résonance pour viser les grands pays qui favorisent le changement climatique dont nous sommes les premières victimes. » (Les Nouvelles de Tahiti, 30/03/2010)

Cette dernière mention illustre une stratégie de victimisation qui permet l'accès à un nouveau type de rente, la rente climatique (Gay, 2014), qui propose le paradoxe suivant : les petits Etats insulaires adoptent le discours du développement durable et rejettent sur les grandes puissances les problèmes environnementaux, bien qu'ils se comportent parfois comme des "chevilles ouvrières de l'ultralibéralisme » (Gay, 2014 : 83), en considérant comme ressources aussi bien la rente climatique que le blanchiment de capitaux, les pavillons de complaisance ou le vente de leurs voix sur la scène internationale. Or, la gestion interne de l'environnement dans ces territoires est très loin d'être exempte de reproches en termes de gestion des littoraux (extraction de sable, remblayage, etc.). De fait, la question du réchauffement climatique, dont l'empirie est encore limitée (Gemenne, 2010) - suscite des controverses, des prédictions alarmistes voire un révisionnisme climato-sceptique hors du champ scientifique, quand bien même le consensus de la communauté scientifique sur un tel constat fait désormais autorité.

Un effet de zoom sur les enjeux relatifs aux transformations environnementales permet d'une part de cerner les différents enjeux politiques, sociaux et économiques de problématiques qui s'imposent à l'agenda politique, et, d'autre part, d'analyser les 
variations des leaderships dans la mobilisation des ressources politiques en Polynésie française.

\section{Les enjeux politiques face à l'ampleur des mutations environnementales}

Le renouvellement des enjeux politiques s'articule avec la démultiplication des transformations environnementales, au sujet desquelles les réponses politiques apparaissent lentes et peu coordonnées face à la complexité des problèmes écologiques et énergétiques.

\section{La multiplicité des transformations environnementales à portée politique}

Le champ du politique est confronté à l'examen de phénomènes qui prennent leurs sources hors du champ des institutions publiques, mais qui appellent une capacité de régulation dans l'espace public, constituant un enjeu politique (Bourg et Whiteside, 2010). Progressivement, la problématique environnementale s'est imposée à l'agenda politique à partir de nouvelles difficultés biopolitiques $^{3}$ : interaction entre la qualité de l'environnement naturel et la santé des organismes vivants, phénomènes climatiques changeants, menaces écologiques, pollutions... Les slogans ou les déclarations d'intentions environnementales des acteurs politiques demeurent insuffisantes et souvent déconnectées du champ de la recherche scientifique pour formuler des réponses englobant les diverses parties de problématiques complexes. Par exemple, comment remédier à la question de la pollution des littoraux sans repenser les modes de consommation ou de gestion de l'urbanisme? En ce sens, les mutations technologiques peuvent être particulièrement utiles à de nouvelles formes d'action publique (Cornu, 2008), en particulier face à la vitesse des transformations environnementales.

Par l'immensité de son étendue maritime, la Polynésie française est confrontée à de nouvelles données environnementales telles que le développement de toutes les formes de pollution, le réchauffement climatique à moyen terme - même si son effectivité et son ampleur restent à déterminer (Gay, 2014) -, ou la convoitise économique des zones de pêche ou d'exploitation minière. En matière de pollution, un des exemples les plus spectaculaires reste la grande poubelle du Pacifique, immense plaque tourbillonnante de déchets flottants dans laquelle la masse de plastique correspond à six fois la masse du plancton (Le Monde, 9/05/2012). La pollution des lagons et des barrières de corail - qui représentent $0,2 \%$ de la surface océanique mais contiennent environ $25 \%$ des espèces marines -, s'ajoute au risque d'une nouvelle pollution nucléaire au regard des fissures dans les atolls de Moruroa et Fangataufa (archipel des Tuamotu), ou, sur le plan " terrestre ", à la gestion des déchets produits dans les îles les plus peuplées. En termes de biodiversité, si la pollution peut à l'évidence menacer la faune sous-marine, la prolifération d'espèces invasives, telles que les plantes envahissantes (Larrue, 2008), pose de nouveaux défis aux autorités publiques ${ }^{4}$. Par exemple, la fourmi de feu (Wasmannia auropunctata) menace ${ }^{5}$ la biodiversité et les activités humaines, notamment dans les lieux d'activité agricole (faapu; apiculture, élevage) ou touristique. Enfin, la pollution de l'océan - la concentration de polluants organiques qui altèrent le fonctionnement des chaînes alimentaires -, de ses habitants et du sol amène à s'interroger sur les enjeux des politiques publiques en matière de santé et économiques en matière de pêche côtière (David, 2008). Outre la question épineuse du financement de la protection sociale, il s'agit de constater prosaïquement que le poisson représente une part importante de l'alimentation insulaire, même si les voies de la nutrition évoluent sous l'influence de la société de consommation (Serra-Mallol, 2007).

\section{Les enjeux politiques du réchauffement climatique}

La double question du réchauffement et des réfugiés climatiques (Mac Adam, 2012; White, 2011 ; Cournil et Mayer, 2014) concernerait en particulier certains atolls de l'archipel des Tuamotu. L'examen de quelques exemples en matière de changement climatique révèle des enjeux politiques communs pour de nombreux territoires insulaires. Le groupe d'experts intergouvernemental sur l'Evolution du Climat (GIEC) rappelle régulièrement aux autorités politiques les impacts potentiels en matière de transformations environnementales à partir de constats scientifiques pluridisciplinaires ${ }^{6}$.

3. La biopolitique, régulation du biologique par le politique, a été analysée comme un mode de gouvernementalité du biopouvoir (Foucault, 1976). Mais l'introduction de la biopolitique, définie en tant que facteur comparatif d'analyse des mutations du politique, met en évidence la façon dont le pouvoir politique agit sur le champ du vivant (corps des individus, milieu naturel). La valeur ajoutée scientifique du concept de biopolitique pour les sciences humaines et sociales résiderait dans le croisement de problématiques et de données scientifiques transdisciplinaires.

4. Le Programme régional océanien de l'Environnement (PROE), entré en vigueur en 1996, possède des compétences pour agir en ce domaine mais n'est doté que de très peu de moyens (Agniel, 2008).

5. L'association Fenua Animalia à Tahiti a alerté en vain les autorités territoriales polynésiennes depuis l'apparition de la fourmi ravageuse au début des années 2000. Elle est désormais recensée par la Direction de l'Environnement parmi les espèces envahissantes (http://www.environnement.pf/spip.php?rubrique53).

6. Un des nœuds de la négociation internationale sur le réchauffement climatique se trouve notamment dans l'opposition entre croissance économique et action contre le changement climatique. Or, les deux s'excluent-ils nécessairement? (voir le $5^{\mathrm{e}}$ rapport du GIEC sur les changements climatiques et leurs évolutions futures, 3 volumes, 2013-2014 (www.ipcc.ch). 
D'autres groupements scientifiques développent des données directement relatives à la question des réfugiés climatiques. Par exemple, l'Internal Displacement Monitoring Centre (IDMC, http:// www.internal-displacement.org), à partir d'un modèle gravitationnel intégrant variables climatiques et facteurs migratoires, élabore différents scénarios migratoires en fonction des types d'impacts du changement climatique variant selon la configuration géographique et humaine des territoires.

La dimension politique de la lutte contre le réchauffement climatique a été mise en lumière par les démarches internationales du président des Maldives Mohammed Nasheed (Gay, 2014), et dans une moindre portée médiatique, par les différents projets afférents aux cas des Kiribati ou des Tuvalu (Joyau, 2008 ; Gemenne, 2010 ; Gay, 2014). Parmi les territoires français, la Polynésie française et la Nouvelle-Calédonie seraient les régions les plus affectées par la hausse du niveau des mers à l'horizon 2100, car elles abritent 60\% des îles menacées (30\% chacune) - entre 6 et $12 \%$ des îles françaises sont concernées. Selon cette étude parue dans Nature Conservation ${ }^{7}$, il s'agit de l'estimation la plus basse, en prenant en compte des îles qui seraient submergées à $100 \%$ en permanence, et non celles perdant 70 , 80 ou $90 \%$ de leur territoire. Cette projection amène à réfléchir sur les enjeux politiques et sociaux qu'un renchérissement des difficultés climatiques produirait sur certaines activités économiques (coprah) ou sur le quotidien des habitants des atolls, déjà en prise avec la gestion de l'eau douce. Selon le biologiste Pierre Gillet, qui pointe particulièrement les risques de multiplication des cyclones, "les Polynésiens pourraient être les premières victimes de la remontée du niveau marin" (Les Nouvelles de Tahiti, 5/11/2010). Toutefois, cela soulève de nouvelles problématiques juridiques concernant le statut des réfugiés climatiques et des Etats potentiellement engloutis (Joyau, 2008; Lallemant-Moe, 2009), ainsi que des réflexions sur les manœuvres d'instrumentalisation politique d'une nouvelle rente climatique. Le cas limite de Tuvalu, dont les causes de la menace de montée des eaux sont quelquefois mal interprétées - même la montée des eaux a pu être remise en cause (Joyau, 2008 ; Lazrus, 2009, 2012 ; Gay, 2014) - met en évidence certaines formes de désinformation, entre l'activisme écologique, le sensationnalisme journalistique et le fonctionnement souvent opaque des Nations unies. Sans se prononcer sur les fondements du débat scientifique relatif à la montée du niveau de la mer - mesurée à 3 millimètres par an à Tahiti entre 1950 et 2009, et entre 2.5 et 2.9 millimètres par an dans certains atolls des Tuamotu-Gambier selon Virginie DuvatMagnan, géographe à l'université de La Rochelle et membre du GIEC - cette thématique n'en reste pas moins un nouveau référent des politiques internationales.

\section{L'environnement face aux enjeux géopolitiques et économiques}

Les questions de l'exploitation et de la gestion des ressources naturelles (nickel en NouvelleCalédonie) et des zones économiques exclusives (Polynésie française et Wallis-et-Futuna) s'inscrivent dans des logiques géopolitiques qui dépassent le cadre du Pacifique océanien par leurs enjeux économiques.

Ainsi, en Polynésie française, "seulement" $40 \%$ de la zone économique exclusive (ZEE) sont exploités pour une surface de 5,5 millions de $\mathrm{km}^{2}$, ce qui représente 6000 tonnes de poissons pêchés à la palangre en 2012 (Les Nouvelles de Tahiti, 14/08/2013). Paradoxalement, les niches économiques potentielles au niveau de la conserverie et de la congélation du poisson sont évoquées par le gouvernement Flosse en 2013. Mais les bateaux de pêche polynésiens ne trouvent pas d'équipage pour partir plusieurs mois en mer. Les autorités territoriales souhaitent donc que la Polynésie devienne éligible en tant que ртом au Fonds européen des affaires maritimes, en contrepartie d'une ouverture de la ZEE à l'Ue, au grand dam des syndicats de pêcheurs professionnels. Ceuxci mettent en avant leur capacité à exercer un mode de gestion traditionnel des aires protégées et des ressources, le Rahui (Bambridge, 2006), ainsi que la nécessité de protéger cette richesse polynésienne, afin, revendiquent-ils, de "ne pas céder notre océan " (Les Nouvelles de Tahiti, 16 août 2013). En particulier, le rôle des puissances asiatiques et surtout de la Chine dans la surpêche mondiale ${ }^{8}$ (extermination des

7. Les scientifiques ont travaillé sur des hypothèses de hausse du niveau de la mer, allant de 1 à 3 mètres autour de 2100 , une fourchette désormais avancée par de nombreuses études. Ils ont croisé les profils de 1269 îles françaises (sur les 2050 existantes de plus d'un hectare) avec ces projections d'élévation des eaux. Selon leurs résultats, entre $6 \%$ (dans l'hypothèse d'une hausse d'un mètre) et $12 \%$ (hypothèse de 3 mètres) des îles françaises sont menacées d'être totalement submergées (La Dépếche de Tahiti, 12/09/2013). Voir les documentaires de John Shenk, Le combat d'un président (2012) à propos des Maldives, et celui de Tom Zubricki, La marée affamée (2011), au sujet de Kiribati.

8. Les navires de pêche chinois ont siphonné, loin de leurs côtes, entre 3,4 millions et 6,1 millions de tonnes de poissons par an entre 2000 et 2011 (64\% en Afrique), dont 0,2 million de tonnes en Océanie. Il s'agirait du premier chiffrage effectué, selon une étude internationale coordonnée par le biologiste Daniel Pauly (université de Colombie-Britannique), publiée dans la revue Fish and Fisheries puis reprise dans la revue Nature. Pékin ne déclarait que 368000 tonnes de poisson en moyenne auprès de la Food and Agriculture Organization (FAO), soit douze fois moins que le chiffrage cité (Le Monde, 4/04/2013). 
requins (Stewart, 2006) ; forte diminution des stocks halieutiques), représente une source de convoitise des ressources océaniques et de l' " or bleu » du Pacifique insulaire.

En outre, "l'or gris" - les ressources des gisements sous-marins - est au cœur d'un enjeu croisé entre l'État, ses territoires et les multinationales du secteur sur la question des nodules polymétalliques, des encroûtements cobaltifères et des "terres rares». En Polynésie française, seuls les encroûtements cobaltiferes présentent pour le moment un réel potentiel, mais une future exploitation dépendra notamment des résultats des expertises en cours demandées par l'État français et le gouvernement polynésien.

Dans le sillage de ces recherches, des enjeux de souveraineté dans la gestion des ressources naturelles ont été mis en évidence par les négociations du programme d'Extension raisonnée du plateau continental (EXTraplac). Initié en 2002, il comprend une déclinaison pour la zone de compétence territoriale (Taglioni, 2007)9. Néanmoins, la question des ressources minérales profondes et demeure un "serpent de mer" récurrent depuis plusieurs décennies, dépendant de capacités technologiques d'extraction et d'exploitation encore lacunaires.

Par ailleurs, des liens entre transformations environnementales et révolution technologique émergent d'initiatives provenant de la société civile. Par exemple, la Polynésie française a été le premier pays insulaire du Pacifique Sud à organiser des conférences indépendantes sur le modèle de TED (Technology, Entertainment, Design), cette organisation de la Silicon Valley (http://www. tedxpapeete.com) qui diffuse les idées par la multiplication de micro-conférences. Dans le secteur économique, les acteurs font naviguer leurs intérêts en fonction des garanties juridiques et de la diminution des risques induits par la présence souveraine de l'État dans les territoires français du Pacifique. L'homme d'affaires Richard Bailey - PDG du groupe Beachcomber - est le promoteur d'une énergie propre et renouvelable mise en place sur l'atoll de Tetiaroa. $\mathrm{Au}$ fait des complications bureaucratiques avec l'administration locale (Montet et Venayre, 2013), et quelquefois néopatrimoniales dans plusieurs endroits du Pacifique insulaire (Murray, 2007), cet entrepreneur économique compte sur l'État :

«Nous comptons beaucoup sur la France. Nous voyons en la France, un garde-fou, un arbitre. Une sécurité très rassurante, pour soutenir le gouvernement autonome de la Polynésie [...]. Notre souhait, c'est d'être l'effet catalyseur de la création d'un véritable pôle de compétitivité en Polynésie autour des énergies renouvelables. » (Sénat, rapport n $\left.{ }^{\circ} 293\right)^{10}$

L'énergie thermique des mers représente une technologie environnementale adaptée à la configuration géographique de nombreuses îles polynésiennes et océaniennes, dès lors qu'un accès à des eaux suffisamment profondes se trouve à proximité de la côte. La Polynésie française est pionnière en la matière, en raison d'une configuration sous-marine favorable grâce à la profondeur de la zone océanographique. Ce dernier exemple illustre l'importance du leadership de la puissance publique dans l'aide aux initiatives de la société civile et surtout dans la définition et la mise en œuvre de politiques publiques transversales. Malgré tout, les leaderships politiques en mesure de conduire une action publique environnementale et, audelà, d'imprégner les imaginaires sociaux de l'importance des enjeux écologiques (Dwyer, 2005), mobilisent essentiellement des ressources politiques à caractère identitaire ou clientéliste dans une perspective de recherche d'une rente providentielle, et n'intègrent en apparence les transformations environnementales qu'à la marge de leurs entreprises politiques.

\section{Variations des leaderships par la mobilisation de ressources institutionnelles, identitaires et rentières}

\section{Le leadership institutionnel de l'État français face aux mutations environnementales}

La redéfinition d'une politique de la France dans le Pacifique s'inscrit toutefois dans la continuité d'une politique de puissance (Mrgudovic, 2008) en termes de sécurité régionale et de politique maritime. Désormais, en relation avec le partenariat de l'Union européenne, cette politique proclamée concerne la transition énergétique (éolien offshore adapté aux zones cycloniques, géothermie, hydrolien maritime ou biomasse), le savoir (Plan haute qualité éducative : favoriser l'accueil des étudiants et la mobilité des personnes), la santé (pôles d'excellence vecteurs de coopération régionale) et l'écologie.

Le développement de nouvelles politiques publiques ciblées sur les transformations environnementales met en évidence la marge de manœuvre de l'État sur l'orientation des

9. Polyplac en Polynésie française. Par ailleurs, la Direction des ressources marines est devenue la Direction des ressources marines et minières en juillet 2013 (Les Nouvelles de Tahiti, 25/05/2013).

10. Le groupe de Bailey est à l'origine du projet afin d'alimenter l'hôtel écologique de Tetiaroa à partir de l'énergie thermique des mers. Ce type de projet, également mis en œuvre dans un hôtel à Bora Bora, concernerait à terme l'hôpital du Taaone à Tahiti afin de réduire le coût de l'air conditionné dans un bâtiment surdimensionné (Les Nouvelles de Tahiti, 26/11/2012). 
politiques publiques et une responsabilité dans la démocratisation des pratiques politiques et économiques en termes environnementaux. Le thème du changement climatique, à travers son versant sur le réchauffement climatique, est de plus en plus porté à l'agenda politique et institutionnel. La formation d'un référentiel des politiques publiques (Muller, 2013), c'est-à-dire une vision du monde, une image cognitive qui inspire une politique publique en structurant les représentations des acteurs définissant et mettant en œuvre une politique environnementale en l'occurrence. Le réchauffement climatique devient un sujet progressivement incontournable des acteurs institutionnels, aussi bien aux niveaux de l'État, du gouvernement et des ministères polynésiens. Sur ce point, la double structure administrative et politique affaiblit les perspectives d'une politique environnementale ambitieuse. La rivalité (Al Wardi, 1998) mais aussi la coordination entre la présidence polynésienne et les ministères, auxquels s'ajoutent les partenaires étatiques - dont les représentants, à l'instar du Haut-commissaire, ne sont la plupart du temps que quelques années sur place - annoncent des complications bureaucratiques importantes, qui nuancent la portée des ressources institutionnelles que l'État serait apte à mobiliser face à des transformations environnementales multiformes. D'autre part, la relation du territoire polynésien à l'État français, dans la continuité des rapports clientélistes entraînés par l'installation du Centre d'Expérimentations du Pacifique (CEP) à partir des années 1960, s'avère relativement paradigmatique des logiques clientélistes et rentières qui scandent les relations franco-polynésiennes. L'éventualité d'une diversification des relations de dépendances et des rentes - le "choix des interdépendances " pour paraphraser la formule de Jean-Marie Tjibaou (1985) - est notamment posée par les partisans de la "décolonisation", qui utilisent la mobilisation identitaire dans une logique de réorientation et de diversification des perspectives de relations clientélistes et rentières (Regnault, 2013).

Bien que son rôle soit reconfiguré par rapport à des processus micro (pouvoirs locaux) et macro (primauté du droit communautaire européen, dérégulation économique, révolution technologique), qui obèrent sa souveraineté et la verticalitédeson pouvoir de décision, l'État possède une latitude d'action dans le développement d'espaces innovants et originaux au sein de la République qui respectent la biodiversité, l'art de vivre de ces territoires et l'approfondissement du savoir-vivre démocratique.

Sur le plan géopolitique, la réaffirmation de "l'enjeu stratégique " de la zone Asie-Pacifique pour la France ${ }^{11}$ par le ministre de la Défense, Jean-Yves Le Drian (Sénat, rapport d'information $\left.\mathrm{n}^{\circ} 293,17 / 01 / 2013\right)$, rappelle que la France ne pouvait se détourner de la région, malgré "les contraintes de la géographie, l'impact de la contrainte budgétaire ou les présupposés erronés sur la dimension économique exclusive que revêtirait la relation euro-asiatique" (Les Nouvelles de Tahiti, 3 juin 2013). Selon le Livre blanc de la Défense et de la sécurité nationale :

«Dans un contexte de tension sur l'accès aux ressources, la France doit être en mesure de marquer sa souveraineté et de défendre ses intérêts dans les outremer » (Les Nouvelles de Tahiti, 4/07/2013)

En outre, le ministre des Outre-mer du gouvernement Ayrault, Victorin Lurel, soulignait que :

" les outre-mer ont vocation à tenir le rôle particulier de relais des intérêts stratégiques de la France" (Les Nouvelles de Tahiti, 7/06/2013)

en relevant les clés d'une nouvelle stratégie. Outre la coopération régionale en lien avec l'Union européenne, le point intéressant de cette approche transformationnelle réside dans le fait de rechercher à " combiner systématiquement " (idem) les intérêts de la métropole avec ceux de ses collectivités, entre prolongement des rapports clientélistes étatiques et ultramarins, et développement d'une logique de coopération. L'ensemble de ces discours, de ces perceptions et représentations renouvelées des autorités françaises mettent en évidence les dynamiques d'une " politique du Pacifique », qui prend forme à partir de divers travaux qualitatifs ${ }^{12}$. Lors du colloque organisé au Sénat en janvier 2013 sur la place de la France dans le Pacifique, les enjeux suivants avaient été mentionnés: protection de la biodiversité, protection et exploitation

11. Selon le vice-amiral Jean-Louis Vichot, lors du colloque sur le Pacifique organisé au Sénat en janvier 2013: «Le Pacifique sera la première source d'emploi pour la France. L'outre-mer sera crucial pour les ressources minérales, alimentaires, biologiques, pour le développement de la recherche au $\mathrm{XXI}^{\mathrm{e}}$ siècle (...). Ce n'est pas la question de l'avenir de la richesse de la France, que l'on développe en Nouvelle-Calédonie, que l'on développe à Wallis-et-Futuna, où on a découvert des encroûtements minéraux d'une rare qualité, et également en Polynésie française où l'on espère beaucoup des progressions de la pêche avec le réchauffement climatique qui n'apporte pas que des catastrophes, puisqu'il va [...] déplacer la ceinture du thon vers la zone exclusive de la Polynésie française " (cité dans Les Nouvelles de Tahiti, 18/11/2013).

12. Outre le colloque au sénat, l'avis du Conseil économique, social et environnemental Pour un renforcement de la coopération régionale des outre-mer en 2012, la conférence internationale organisée par le CESE intitulée : «Haute mer, avenir de l'Humanité, quelle gouvernance pour une gestion durable de l'océan ?' ", afin de peser sur les négociations des Nations unies, qui doivent aboutir en 2014 à un cadre international pour la gestion de la biodiversité en haute mer (La Dépêche de Tahiti, 12/04/2013). 
des données halieutiques, développement économique, nodules polymétalliques et extension du domaine continental. Ainsi, la " combinaison systématique des intérêts" tente de répondre à un point important soulevé par l'historien Jean-Marc Regnault: des conflits futurs peuvent naître d'intérêts opposés entre les territoires du Pacifique et ceux de l'État ${ }^{13}$. Malgré tout, selon l'ancien Ambassadeur de la France dans le Pacifique, Hadelin de La Tour du Pin, la France maintient son intérêt pour le Pacifique :

«Donc il est évident que tant que nos concitoyens français du Pacifique souhaiteront rester français, la France restera avec eux. Nous mettrons les moyens nécessaires à leur prospérité, à leur stabilité, à leur démocratie $[\ldots]$. Dans une vingtaine d'années, vous aurez une nouvelle génération de leaders politiques, d'électeurs, d'acteurs économiques et il est assez naturel, que les uns et les autres puissent envisager des évolutions auxquelles peut-être nous ne pensons pas encore aujourd'hui. " (Les Nouvelles de Tahiti, $18 / 01 / 2013)$

En outre, la dimension internationale de la question climatique prend un relief intéressant en Polynésie française au regard des enjeux de souveraineté et des évolutions de la configuration institutionnelle des relations entre la France et la Polynésie française. En effet, la négociation du volet "réfugiés climatiques" nécessite une clarification sur la double échelle territoriale et nationale quant au degré de ressources stratégiques disponibles en matière géopolitique et de développement des relations internationales (Gemenne, 2009). Si l'État conserve la compétence régalienne en matière de politique étrangère, la Polynésie française dispose dans son statut d'outils d'affirmation d'une " diplomatie " polynésienne dans la région océanienne.

D'ailleurs, un regard comparatif entre les territoires français du Pacifique Sud sur la question climatique (Worliczek, 2013) amène à envisager des convergences institutionnelles dans le champ des politiques publiques relatives à l'environnement, à partir de données anthropologiques et sociologiques pertinentes. La confrontation de ces problématiques avec le politique est illustrée dans la manifestation des leaderships politiques.

\section{Un leadership politique axé sur la mobilisation identitaire}

L'entreprise identitaire de la revendication d'indépendance défendue par le leader du Tavini Huiraatira, Oscar Temaru, vise à transformer la Polynésie française en Maohi Nui dans une démarche politique inscrite dans une forme de messianisme religieux du souverainisme (Saura, 2008). La question du "pouvoir de l'identité " (Castells, 1999) se traduirait à Tahiti par une " obsession de la France» (Al Wardi, 2008), face à laquelle les préoccupations environnementales paraissent moins audibles.

La défense d'un "paradis communautaire " (Castells, 1999) repose sur un prisme identitaire voire ethnique de la conception mäohi (Saura, 2008). Elle exprime une réponse-refuge face aux nombreuses dérégulations engendrées par la mondialisation, en laissant parfois de côté les identités-mixtes complexes (Appadurai, 2001). Or, selon l'historien Henry Laurens, le choc des civilisations peut être empêché en admettant " partout la présence de l'autre dans l'identité », de " desserrer l'étau des exclusivismes identitaires", et de "se souvenir qu'un seul individu peut posséder des identités multiples, et toutes contextualisées" (Laurens, 2010). Ces remarques sont d'autant plus pertinentes à l'heure de la révolution numérique, qui efface les barrières structurelles, l'éparpillement géographique des îles, facilite les échanges et les accès à la connaissance par le moyen du câble Honotua - hono, le lien; tua, le large (Argounès et al., 2011: 56) -, qui permet aussi l'individualisation des moyens de production et d'expression dans une "société en réseaux" (Castells, 1998).

En fait, l'hybridité des cultures politiques française et polynésienne (Bessard, 2013) renvoie d'un certain côté à l'analyse anthropologique de Michel Panoff, qui évoquait une "société euro-tahitienne » au sujet de la Polynésie française, car, "[...] c'est le métissage entendu au sens biologique et au sens culturel qui a été le moteur de l'histoire vraiment singulière de Tahiti et des îles voisines" (1986: 230). Le sociologue Bruno Saura (2008 : chap. 7 relatif à "l'idéologie polynésienne ") est depuis revenu sur les limites de l'apologie du métissage par rapport à la perception identitaire mäohi des Tahitiens. Néanmoins, les risques sociaux d'une traduction ethnique voire raciale du message politique ne découragent pas la démarche politique particulière de certains entrepreneurs politiques (Panoff, 1989 : 231). Pourtant, dès les années 1980 , le recours politique à la ressource identitaire pouvait déjà apparaître comme une impasse :

«C'est précisément quand la panmixie est à jamais consommé, que certains politiciens et intellectuels de Papeete découvrent l'ethnicité et veulent ressusciter les valeurs ethniques de la Polynésie traditionnelle

13. «Automatiquement les territoires vont prendre des compétences, et au moment où ils exerceront ces compétences, ils vont forcément entrer en conflit avec les compétences étatiques [...]. Quels sont les intérêts qu'il faut faire passer en priorité ? Sont-ce les intérêts de l'État, ou sont-ce les intérêts des territoires ? "(cité dans Les Nouvelles de Tahiti, 18/01/2013). 
[...]. Or s'il existe une identité tahitienne, elle est à chercher dans le présent et l'avenir, mais certainement pas dans le passé. » (Panoff, 1986 : 231)

L'économie polynésienne repose de façon conséquente sur le protectionnisme et les transferts financiers de métropole (Poirine, 2011). Or, la micropolitique polynésienne n'endigue pas les macro-flux mondiaux, en raison d'une dépendance importante en ressources énergétiques, économiques ${ }^{14}$ et militaires (Duranthon, 2011). Malgré tout, Oscar Temaru a encouragé une réflexion sur l'avenir lors de la réunion du Club de Madrid à Tahiti (Les Nouvelles de Tahiti, 3/07/2012). Sur invitation du président Temaru, avec l'aide du milliardaire australien Clive Palmer, des personnalités se sont jointes aux membres du gouvernement polynésien pour examiner la place du Pacifique dans le Xxi ${ }^{\mathrm{e}}$ siècle. Ensuite, la démarche onusienne du leader indépendantiste s'est inscrite dans une logique de reconnaissance du processus de décolonisation (Regnault, 2013). Sanctionné dans les urnes par ses insuffisances gouvernementales au profit de Gaston Flosse et du Tahoeraa Huiraatira en avril 2013, le leadership politique de Temaru n'en développe pas moins une dimension prospective, qui est présente dans ses discours politiques à connotation identitaire et dans les allusions à des mannes économiques providentielles ${ }^{15}$. Ainsi, la dernière expérience gouvernementale pendant deux ans a permis au parti de réfléchir à de nouveaux objectifs pour les politiques maritimes et de la pêche ("Le Māohi doit renouer avec son océan », http://www.tavinihuiraatira.com).

Le thème des transformations environnementales n'a pas été de grande importance lors de la campagne électorale de 2013, face à la crise économique et sociale. Le Tahoerad Huiraatira a depuis longtemps intégré la question environnementale à son programme, et mis en avant quelques réalisations sur son site Internet (http://www.tahoeraahuiraatira.pf). Dans l'espace politique polynésien, l'écologie ne dispose ni d'une implantation partisane ni d'une audience électorale conséquentes. Peu influent au sein de la coalition Union pour la démocratie (UPLD) emmenée par le Tavini, le parti écologiste Heuira-les Verts de Jacky Bryant, ministre de l'Environnement du gouvernement Temaru (2011-2013), ne possède qu'une place marginale, en raison de son manque de ressources politiques en termes de clientélisme et d'implantation partisane dans les archipels. Le chef du parti écologiste déplore que le président du Tavini "refuse l'expression démocratique qu'il a pourtant initié " (La Dépêche de Tahiti, 20/04/2015) - en référence au Taui ("changement, alternance") de 2004, qui avait vu Temaru conduire la plateforme d'opposition de l'upld. La question environnementale ne constitue pas non plus une préoccupation majeure du leadership identitaire : " on peut s'interroger ", selon Jacky Bryant, sur la sensibilité environnementale d'Oscar Temaru.

En effet, les formes du leadership politique en Polynésie française incluent la prise en compte des mécanismes de réciprocité et de redistribution des ressources matérielles et symboliques (Robineau, 1978, 1985). Le clientélisme est vécu comme une institution sociale, qui alimente des pratiques de courtage encouragées par la structure d'une économie rentière depuis l'installation du Centre d'expérimentations du Pacifique en 1965, à laquelle a succédé la pérennisation des dotations de l'État, acteur fondamental, même indirectement, de l'économie polynésienne (Poirine, 2011). L'idée de "rente nucléaire» imprègne toujours l'économie politique polynésienne. Ainsi, les stratégies des leaders, à la recherche de rentes, continuent d'activer les registres du clientélisme interne - les aides de Paris - et international, entre le besoin d'une clientèle onusienne (Regnault, 2013) et le développement d'une diplomatie polynésienne avec les puissances asiatiques (Bessard, 2013) .

La quête d'une rente providentielle pour assumer la conduite d'un État souverain sous Gaston Flosse n'a pas empêché le leadership à résonance identitaire de recourir à des pratiques clientélistes (Al Wardi, 2008). Les pratiques et les ressorts des leaderships politiques polynésiens transcendent alors les clivages partisans quant à la diversification des rentes économiques, sans s'accorder nécessairement sur la provenance de la manne providentielle ni sur la trajectoire de développement (Magrin, 2013). Si les indépendantistes se retranchent derrière le passé colonial et nucléaire en regardant un horizon souverain, les autonomistes, conscients des liens établis et des ressources apportées continuellement par la France, écartent davantage de leur spectre d'action politique la question identitaire au profit d'une orientation programmatique vers des formes plus ou moins

14. Communiqué de Standard and Poor's, Agence Tahitienne de Presse (22/05/2011). Le classement de la Polynésie française au même niveau que la Grèce en 2010 (BB+), par cette critiquable agence de notation, montrait malgré tout les fragilités éco-financières du Fenua (trésorerie exsangue et faibles capacités d'investissement).

15. En 2006, Oscar Temaru se rend en Californie, afin de rencontrer le gouverneur Schwarzenegger pour vendre de l'hydrogène aux automobilistes californiens, mais le projet n'aboutit pas (Les Nouvelles de Tahiti, 30/05/2011). La même année, le leader polynésien déclare : " chaque fois que nous parlons d'être indépendants, de l'autre côté le gouvernement français dit que nous n'aurons jamais assez d'argent pour cela. Notre indépendance n'a pas de prix. Le noni [fruit médicinal] nous aidera à être libres; maintenant, je peux rentrer à Tahiti et dire aux gens, comme Martin Luther King : j’ai observé et j'ai vu la terre promise " (Les Nouvelles de Tahiti, 30/05/2011). 
subventionnées de développement économique. Ainsi, la "virtuosité " des acteurs politiques consiste à se plaindre d'une dépendance en demandant paradoxalement et régulièrement de l'augmenter (Panoff, 1986). Les présidences de Flosse jouaient sur le registre identitaire - en partie pour récupérer la mouvance autonomiste des années 1970 - tout en exigeant toujours plus de subsides de la part de l'État (Al Wardi, 1998 ; Bessard, 2013).

Le leadership de Gaston Flosse et du Tahoeraa Huiraatira développe une approche prospective qui relève davantage de la recherche d'une indépendance économique et d'une autonomie institutionnelle que d'une émancipation politique radicale. L'intérêt de la question environnementale prend en particulier la forme du développement de la perspective d'une rente minière ou climatique.

\section{Un leadership politique en quête de rentes, clima- tique ou autres...}

Dans une relation complexe à l'État français selon une logique de «partage du pouvoir " (Al Wardi, 1998), la domination politique du leadership flossien entre 1982 et 2004 a mis en évidence la conception d'un développementalisme rentier (Bessard, 2013), c'est-à-dire un système économique qui combine les avantages structurels d'une économie de rente défiscalisée entretenue par la France et le développement des potentialités économiques polynésiennes (tourisme, perliculture, vanille, coprah, etc.) en vertu d'une idéologie qui place le gouvernement local comme acteur majeur de l'économie (Montet et Venayre, 2013), à la limite du chevauchement entre ressources publiques et privées (straddling). Dans ce contexte, la question environnementale n'apparaît qu'à la marge des politiques publiques territoriales.

En 1984, après l'obtention du statut d'autonomie, Gaston Flosse déclarait qu'il s'agissait de «tendre vers l'indépendance économique ", dans laquelle il voyait un "dessein susceptible de mobiliser les efforts et l'enthousiasme de notre pays. " (Bessard, 2013: 240). Le jeune député chiraquien, qui érigeait le pouvoir de décision dans le domaine économique comme une priorité, était alors à l'origine de l'article 62 (évoqué dans le projet de loi d'autonomie de 1980), qui prévoit des possibilités d'exploration, de recherche et d'exploitation de toutes les ressources biologiques et minérales dans la ZEE, mais aussi d'autorisation d'investissements étrangers en-deçà d'un certain seuil. Après le retour au pouvoir de « Papa Flosse » en avril 2013, plusieurs champs d'activités économiques ont été ciblés: pêche, aquaculture, hub touristique, technologique (numérique) ${ }^{16}$ et scientifique (environnement et fonds marins, "terres rares"). Certains chantiers s'inscrivent dans la reprise de projets du précédent gouvernement (aquaculture, question minière : modification du code minier) ${ }^{17}$. Toutefois, un effet d'accélération est mis en évidence par plusieurs projets du gouvernement Flosse relatifs aux dossiers de "l'or bleu " et de "l'or gris".

Le leadership relatifà ces secteurs est notamment impulsé par le ministre des Ressources marines, des Mines et de la Recherche, Tearii Alpha, qui multiplie les rencontres à Paris et à Bruxelles, pour promouvoir une "plate-forme internationale de recherche", et "faire de la Polynésie une terre d'innovation et de recherche" (Les Nouvelles de Tahiti, 14/06/2013). Le ministre polynésien met la France et l'Union européenne au pied du mur ("on n'a pas vingt ans devant nous»), en menaçant implicitement d'un basculement de réseaux d'intérêts en faveur des Chinois, des Japonais ou des Canadiens :

" ça intéresse ceux qui seront capables d'aller sous l'eau. Nous pensons que cela doit intéresser en premier la France et l'Union européenne. " (Les Nouvelles de Tahiti, 14/06/2013)

Mais, s'ils ne sont pas réactifs, le ministre annonce que d'autres puissances viendront, car :

"Les Russes investissent le Pacifique, les Chinois et les États-Unis aussi. " (Les Nouvelles de Tahiti, $14 / 06 / 2013)$

L'exploitation n'interviendra pas, au regard des technologies actuelles, avant vingt ou trente ans. Cette démarche s'inscrit selon ses promoteurs dans une réflexion pour recréer une activité économique à long terme et "tourner définitivement la page " des essais nucléaires :

«Nous avons contribué pour la Défense nationale, on souhaiterait contribuer au développement des technologies du futur au travers de ce potentiel minier. "

Le hub scientifique international concernerait les écosystèmes tropicaux et insulaires, les ressources marines et maritimes, les énergies renouvelables et la biotechnologie, avec l'implantation d'une unité mixte internationale avec le réseau du CNRS en Polynésie française, qui constituerait, selon le ministre, "un atout important pour atteindre les objectifs de la

16. Un Observatoire de l'économie numérique a été créé en mai 2014 et mis en place le 25 février 2015.

17. L'ancien ministre de l'Environnement du gouvernement Temaru, Jacky Bryant, a ainsi demandé au nouveau gouvernement Flosse de reprendre le Plan climat-stratégie (140 orientations à mettre en place à court, moyen et long terme), même " peint en orange ", c'est-à-dire la couleur du parti politique de G. Flosse (Les Nouvelles de Tahiti, 18/09/2013). 
politique maritime intégrée de la Polynésie française. "(Les Nouvelles de Tahiti, 22/07/2013).

$\mathrm{La}$ dimension transformative $\mathrm{du}$ leadership politique Gaston Flosse, qui a développé une «certaine idée de Tahiti Nui " selon l'expression de Jean-Marc Regnault (2003), apparaît tout au long de sa carrière, en tant que "créateur" d'institutions (commune de Pirae, statuts d'autonomie; secrétariat d'État au Pacifique Sud). Toutefois, Gaston Flosse "absorbe tous les projets", qu'ils relèvent du développement économique classique ou de secteurs innovants, selon différents observateurs de la scène économique tahitienne. Le leader agit en fonction d'une logique d'entreprise politique, qui le situe davantage dans une perspective classique et transactionnelle que dans une logique transformationnelle (Bessard, 2013). "Il ne s'agit pas d'innover, d'inventer", déclarait le président de la Polynésie française, mais d'appliquer le programme du parti (Journal télévisé, Polynésie 1ere, 9/09/2013).

Pourtant, l'approfondissement des relations avec la Chine illustre la quête de ressources économiques et politiques du leader polynésien, quiavait ouvert lavoieà des relations commerciales avec Pékin dans le sillage des réseaux chiraquiens. Les demandes de financement à des organismes bancaires chinois, l'installation d'une société chinoise dans l'aquaculture (poissons à haute valeur ajoutée sur le marché chinois : mérous, loches, coquillages) dans l'atoll de Hao (archipel des Tuamotu), en contrepartie notamment de l'embauche d'une main-d'œuvre polynésienne, ou la proposition à différents investisseurs étrangers dans le projet du Mahana Beach (complexe touristique à Punaauia, Tahiti), ainsi que l'étude du modèle hawaïen pour le tourisme (La Dépêche de Tahiti, 27/07/2013) mettent en exergue la quête de développement économique et de nouvelles mannes financières, en évacuant la problématique environnementale derrière l'impératif financier et touristique.

Cependant, la question des changements climatiques, au coeur des transformations environnementales, fait de plus en plus l'objet d'une préoccupation politique au niveau territorial. Par exemple, les atolls de Napuka et de Puka Puka dans l'archipel des Tuamotu ont connu une perte de $90 \%$ de la production de coprah de la cocoteraie, menacée par l'océan, ainsi que l'a signalé en conseil des ministres par le ministre de l'Agriculture Thomas Moutame (Les Nouvelles de Tahiti, 12/08/2013) ${ }^{18}$. Tauira Puarai, le maire de Napuka, a fait part de son inquiétude aux autorités polynésiennes, car l'activité de l'île est impactée. Dans un discours explicite sur la question climatique, le président Flosse a estimé que :
«La montée des eaux n'est pas encore très significative, mais il faut prévoir l'avenir [...]. Il y a des terres aux Marquises ou dans d'autres îles, mais nous n'en sommes pas encore là. » (Les Nouvelles de Tahiti, $26 / 08 / 2013)$

De plus, cette thématique a également été mobilisée par le leader polynésien lors du 44e Forum des îles du Pacifique aux îles Marshall. Gaston Flosse proposait de créer une fondation mondiale pour permettre la réinstallation des populations exposées à la montée des eaux, évoquant même la possibilité pour la Polynésie de s'ouvrir aux réfugiés climatiques. Or, pour l'ancien ministre Jacky Bryant, il s'agit d'une compétence de l'État en matière d'accueil de populations étrangères :

" ce n'est pas une compétence de la collectivité et encore moins de Gaston Flosse. [...] Plutôt que d'avoir la modestie de proposer une démarche très constructive, il s'est embarqué dans des déclarations laissant presque croire qu'il serait le président d'un État indépendant. »(Les Nouvelles de Tahiti, 26/08/2013)

Outre le prestige politique conféré dans la région par l'esquisse d'une diplomatie polynésienne d'un "presque chef d'Etat", l'intérêt pour la rente climatique fait aussi résonner la promesse d'un autre levier de ressources pour la Polynésie française. Ainsi, le député Jean-Paul Tuaiva demandait au gouvernement de Manuel Valls des "moyens financiers " supplémentaires pour faire face aux changements climatiques et à la transition énergétique, lors de la séance à l'Assemblée nationale le 13 mai 2015. La ministre de l'Écologie, du Développement durable et de l'Énergie, Ségolène Royal, a rappelé les efforts de l'État dans l'accompagnement du territoire vers les énergies renouvelables et le financement de projets en cours, par exemple la climatisation de l'hôpital du Taaone à Tahiti.

Les mannes successives (présence américaine à Bora pendant la Deuxième Guerre mondiale, coprah et phosphate après-guerre, tournage des Mutinés du Bounty en 1961, construction de l'aéroport, CEP, perle noire, défiscalisation, dotation de l'État) encouragent les leaders polynésiens à mettre régulièrement en avant les nouvelles potentialités, et dorénavant les risques, de l'avenir d'un territoire de 270000 habitants, mais sans remettre en cause les rentes de situation qui fondent de fortes inégalités sociales à Tahiti (Regnault et Al Wardi, 2011 : 108-117). Dès lors, le salut économique, en-dehors de la mise en valeur des ressources locales, passe une nouvelle fois par la pérennisation des dotations de Paris à Tahiti - par exemple via le Contrat de

18. À Napuka, le phénomène de dégénérescence de la cocoteraie est accentué par la présence d'une maladie véhiculée par un insecte nuisible, le Brontispa longissima, surnommé la peste du cocotier. 
Projets État-Polynésie française (2015-2020), qui prévoit un volet sur les énergies renouvelables -, des demandes d'avance de trésorerie à l'État, face à la difficile situation financière du territoire ${ }^{19}$. En 2013, selon l'Institut d'Emission d'OutreMer (IEOM), l'État a dépensé 176,6 milliards de FCFP (1,48 milliards d'euros), soit $23 \%$ du PIB de la Polynésie française dans l'économie locale, comprenant les salaires versés aux fonctionnaires, les dépenses de fonctionnement et d'investissement, les retraites et les transferts aux collectivités territoriales. Cependant, le leadership politique continue de s'exercer dans la logique d'une mise en œuvre extensive des prérogatives du statut d'autonomie.

Selon le président de la Polynésie française Edouard Fritch, président-délégué du Tahoeraa, ancien président de l'Assemblée de la Polynésie française et gendre de Gaston Flosse, qui a pris la succession de ce dernier - non d'ailleurs sans conflits qui persistent jusqu'à aujourd'hui entre les deux clans respectifs :

"L'autonomie est le meilleur outil qui nous permet d'être nous-mêmes, d'être ouverts sur un monde globalisé, d'être maîtres de nos choix économiques, fiscaux et sociaux et d'être fiers de notre identité ; tout cela à condition bien entendu d'être en capacité et d'avoir les compétences pour utiliser les multiples ressources institutionnelles de notre statut. " (Les Nouvelles de Tahiti, 20/09/2013)

La complexité des enjeux relatifs aux transformations environnementales semble laisser les leaderships politiques polynésiens dans l'expectative, pris par les impondérables enjeux de réélection et de gestion des rapports de force politique locaux.

\section{Conclusion}

Face à la question des transformations environnementales, la France a d'un côté les mains liées par les différents transferts de compétences, tandis que de l'autre, les enjeux environnementaux sont appréhendés par les notables politiques dans une logique de réappropriation de nouveaux projets, par exemple ceux liés à la politique européenne.

Le passage des préoccupations environnementales dans le champ politique, en dépit des limites et des faiblesses de la parole politique (Latour, 2002), montre que ces sujets ne peuvent demeurer dans l'entre-soi scientifique en raison des enjeux démocratiques sous-jacent. Ce constat appelle à concevoir le collectif des hommes et de la nature, en partie dans le sens quel'anthropologue Bruno Latour tâche de redonner à l'écologie "politique " (Latour, 1999). Mais, dans le cas polynésien, les compétences du pouvoir local conféré par le statut d'autonomie politique aux côtés d'une puissance économique et militaire française, laissent perplexes quant à l'abandon d'une protection politique et économique dans les macro-flux planétaires, en considérant derechef les limites des gouvernementalités postcoloniales face au développement durable (Li, 2007). En outre, il subsiste une contradiction entre une volonté d'émancipation institutionnelle et la perte des compétences de l'État, qui possède les capacités techniques et transformatives indispensables aux territoires français du Pacifique face aux changements climatiques. Pour autant, ces domaines transformationnels révèlent une source d'intégration entre les entités françaises $d u$ Pacifique Sud et leur intégration dans la région. À condition que les leaderships politiques territoriaux en Polynésie française considèrent l'intérêt d'aborder les transformations en coopération avec une puissance étatique oscillant entre l'oubli de ses Outre-mers et la reconsidération de la question Pacifique.

\section{BIBLIOGRAPHIE}

Agniel Guy, 2008. La gestion de l'environnement dans le Pacifique. Moyens et objectifs, Journal de la Société des Océanistes 126-127: L'environnement dans la Pacifique Sud, J. Trichet et I. Leblic (éds), pp. 145-151 (http:// jso.revues.org/3842).

\section{Al Wardi Sémir, 2008. Tahiti Nui ou les dérives} de l'autonomie, Paris, L'Harmattan, 263 p.

19. En 2010, les dépenses de l'État s'élevaient à 179 milliards de FCFP (soit 1,5 milliard d'euros ; statistique ISPF), pour 270000 habitants en 2011. Après une carrière effectuée dans les cabinets des gouvernements successifs de Flosse, Temaru et Tong-Sang, Maïana Bambridge estime, au sujet des années Flosse, que "Ce monsieur a pu tirer profit de la manne nucléaire mais n'a pas pu construire une économie durable». D'autant que l'instabilité politique post-taui (alternance en 2004-2005 marquée par la chute du gouvernement Flosse) a nourri un climat économique maussade marqué par le déclin de l'emploi salarié et le déficit de la balance commerciale. Une autre explication sur l'absence de transformation économique est fournie par un militant et agriculteur, qui a travaillé pour l'onu, Gabriel Tetiarahi : "J'ai choisi de ne pas travailler avec des gouvernements ici [...] à cause de la corruption [...]. De plus, nos gouvernements successifs n'ont eu aucune vision de politique de production pour la transformation et l'exportation de nos produits [...]. On s'en rend compte avec le gouvernement indépendantiste [...]. C'est difficile de travailler avec des équipes qui n'ont aucune notion de ce qu'est une politique agricole. Ils n'ont aucune notion de ce qu'est un plan stratégique. La situation est bloquée [...]. Le gouvernement décide trop de politiques à mettre en place sans consulter les professionnels des secteurs concernés. Ici, les politiques économiques sont faites pour bénéficier aux familles politiques. " (Les Nouvelles de Tahiti, 21/03/2013). 
Al Wardi Sémir et Jean-Marc Regnault, 2011. Tahiti en crise durable. Un lourd héritage, Papeete, Éd. de Tahiti, 127 p.

Arendt Hannah, 1989 (1972). La crise de la culture, Paris, Folio, 384 p.

—, 1995. Qu'est-ce que la politique?, Paris, Seuil, $195 \mathrm{p}$.

Argounès Fabrice, Sarah Mohamed-GaILLARD et Luc VACHER, 2011. Atlas de l'Océanie. Continent d'îles, laboratoire du futur, Paris, Éd. Autrement, 80 p.

Appaduraï Arjun, 2001. Après le colonialisme. Les conséquences culturelles de la globalisation, Paris, Payot, 322 p.

Balandier Georges, Anthropologie politique, Paris, puf, 1967, 240 p.

Bambridge Tamatoa, 2006. Fondements et évolution du pluralisme juridique en Polynésie française : l'exemple du foncier, in Élise Huffer et Bruno Saura (éds), Tahiti, Regards intérieurs, Suva (Fiji), University of the South Pacific, pp. 29-59.

BAmbridge Tamatoa et Bernard Rigo (éds), 2012. Le Pacifique et la mondialisation, CNRS Éditions, 233 p.

Bass Bernard M. and Ronald E. Riggio, 2006 ( $2^{\text {nd }}$ ed.). Transformational Leadership, Mahwah, NJ, Lawrence Erlbaum Associates.

Bessard Rudy, 2013. Pouvoir personnel et ressources politiques. Gaston Flosse en Polynésie française, thèse de Science politique, sous la dir. de Daniel Bourmaud, Université de Pau, 810 p. (http://www.theses.fr/2013PAUU2011).

Bonneuil Christophe et Jean-Baptiste Fressoz, 2013. L'événement Anthropocène, la Terre, l'histoire et nous, Paris, Seuil.

Bourg Dominique et Kerry Whiteside, 2010. Vers une démocratie écologique. Le citoyen, le savant et le politique, Paris, Seuil.

Braud Philippe, 2004 (7 $7^{\mathrm{e}}$ éd.). Sociologie politique, Paris, LGDJ.

Budoc Rémy Louis, 2012. Pour un renforcement de la coopération régionale des Outremer, Conseil économique social et environnemental, JORF.

—, 2013. Les Outre-mer et l'international: quelle place dans le monde globalisé ?, Paris, Éditions Publisud.

Burns James, 2010 (1978). Leadership, New York, Harper \& Row, 544 p.
-, 2007 (2003). Transforming Leadership, Grove/Atlantic, Incorporated, 336 p.

Castells Manuel, 1998 et 1999. La société en réseaux et Le pouvoir de l'identité, Tome I \& II, $535 \mathrm{p}$.

Casilli Antonio, 2010. Les liaisons numériques. Vers une nouvelle sociabilité ?, Paris, Seuil, 331 p.

Cardon Dominique, 2010. La Démocratie Internet. Promesses et limites, Paris, Seuil.

Celentano Alexandrine Brami, 2002. Frontières ethniques et redéfinition du cadre politique à Tahiti, Hermès 32-33, pp. 367-377.

Cornu Jean-Michel, 2008. Nouvelles technologies, nouvelles pensées?, Paris, FYP.

Cournil Christel et Benoît Mayer, 2014. Les migrations environnementales, Paris, Presses de Sciences Po, $120 \mathrm{p}$.

David Gilbert, 2008. La pêche côtière océanienne et son environnement. La dialectique instabilité/stabilité au fil de l'eau, Journal de la Société des Océanistes 126-127: L'environnement dans la Pacifique Sud, J. Trichet et I. Leblic (éds), pp. 247-270 (http://jso.revues. org/4352).

De Deckker Paul, 2002. Le Pacifique à la recherche du développement dans un espace émietté, Revue française d'administration publique 101, pp. 157-168.

Demmer Christine et Marie Salaün (éds), 2006. À l'épreuve du capitalisme: Dynamiques économiques dans le Pacifique, Paris, L'Harmattan, $200 \mathrm{p}$.

Duranthon Arnaud, 2011. Quelle souveraineté pour les micro-États d'Océanie ?, in J.-Y. Faberon, V. Fayaud et J.-M. Regnault (éds), Destins des collectivités politiques d'Océanie: Peuples, populations, nations, Etats, territoires, pays, patries, communautés, frontières, Presses universitaires d'Aix-Marseille (PUAM), 2 vol., pp. 499-508.

Duvat-Magnan Virginie, 2012. Les Outre-Mer face au défi du changement climatique, contributeur principal, ONERC, rapport au Premier ministre et au Parlement, Paris, La Documentation française, $216 \mathrm{p}$.

Dwyer D. Peter, 2005. Ethnoclassification, Ethnoecology and the Imagination, Journal de la Société des Océanistes 120-121: Ethnoécologie en Océanie, pp. 11-25 (http://jso.revues. org/321).

Favre Pierre, 2008. Ce que les science studies font à la science politique, Revue française de science politique (RFSP) 58, 5, pp. 817-829 
(www.cairn.info/revue-francaise-de-sciencepolitique-2008-5-page-817.htm).

GAY Jean-Christophe, 2014. Réchauffement climatique : l'instrumentalisation des îles, in L'Espace géographique 43, 1, pp. 81-89 (http:// www.mgm.fr/ARECLUS/page_auteurs/ Gay77.pdf).

Gemenne François, 2009. Géopolitique du changement climatique, Lieu ?, Armand Colin, 256 p.

—, 2010. Tuvalu, un laboratoire du changement climatique? Une critique empirique de la rhétorique des 'canaris dans la mine', TiersMonde 204, pp. 89-108 (http://www.cairn. info/revue-tiers-monde-2010-4-page-89. htm).

Hau'Ofa Epeli, 2013 (2008). Notre mer d'îles [trad. de Our Sea of Islands], Tahiti, Pacific Islanders Ed., 40 p.

Hibou Béatrice, 2011. Anatomie politique de la domination, Paris, La Découverte, 298 p.

Joyau Marc, 2008. Tuvalu ou l'infortune de mer, Annuaire de Droit maritime et océanique XXvi, pp. 175-189.

Laburthe-Tolra Philippe et Jean-Pierre WarNIER, 2003. Ethnologie, Anthropologie, Paris, PUF, Quadrige.

Lallemant-Moe Hervé Raimana, 2009. L’apatride climatique et la disparition d'État dans le Pacifique Sud, Revue juridique polynésienne 15, pp. 77-93 (http://www.upf.pf/IMG/ pdf/07-RJP15-L_Apatride-climatique.pdf).

LARrue Sébastien, 2008. Les plantes envahissantes en Polynésie française: un exemple d'approche de la complexité en science de l'environnement, Vertigo 8-2, 14 p.

LATOuR Bruno, 2002. Et si on parlait politique? Politix 15-58, pp. 143-165 (http://www.persee.fr/web/revues/home/prescript/article/polix_0295-2319_2002_num_15_58_1003).

—, 2004 (1999). Politiques de la nature: comment faire entrer les sciences en démocratie, $\mathrm{Pa}-$ ris, La Découverte, $382 \mathrm{p}$.

—, 2008. Pour un dialogue entre science politique et science studies, RFSP 58, 4, pp. 657-678 (http://www.cairn.info/revue-francaise-descience-politique-2008-4-p-657.htm).

Laurens Henry, 2010 (7-13/08). Entretien in Marianne.

Lazrus Heather, 2009. The Governance of Vulnerability: Climate Change and Agency in Tuvalu, South Pacific, in S. A. Crate and M. Nuttall, Anthropology and Climate Change, pp. 240-249.
-, 2012. Book review of Climate change and small island states: Power, knowledge and the South Pacific by Jon Barnett and John Campbell, Pacific Affairs 85, pp. 241-243.

Levine Stephen, 2009. Pacific Ways. Government and Politics in the Pacific Islands, Victoria, Victoria University Press, 302 p.

LI Tania, 2007. The Will to Improve, Duke University Press, 392 p.

MaC ADAM Jane, 2012. Climate Change, Forced Migration and International Law, Oxford University Press.

Magrin Géraud, 2013. Voyage en Afrique rentière. Une lecture géographique des trajectoires $d u$ développement, Paris, Publications de la Sorbonne, coll. Territoires en mouvements, $424 \mathrm{p}$.

Massau Serge, 2010. Paroles d'un autochtone, Papeete, Haere Po, 161 p.

Mazzucato Mariana, 2011. The Entrepreunarial State, London, Demos London, 149 p.

Milacic Slobodan (éd.), 2006. La démocratie représentative devant un défi historique, Bruxelles, Bruyants, 294 p.

Mohamed-gaillard Sarah, 2010. L'archipel de la puissance? La politique de la France dans le Pacifique Sud de 1946 à 1998, Bruxelles, Berlin, New York, Éditions P.I.E. Peter Lang, $428 \mathrm{p}$.

Montet Christian et Florent Venayre, 2013. La concurrence à Tahiti. Une utopie ?, Papeete, Au Vent des îles, 310 p.

Morin Edgar, 2007. Vers l'abîme, Paris, L'Herne, $181 \mathrm{p}$.

Mrgudovic Nathalie, 2008. La France et le Pacifique Sud. Les enjeux de la puissance, Paris, L'Harmattan, $440 \mathrm{p}$.

Muller Pierre, 2013. Les politiques publiques, Paris, PUf, Que sais-je ?, 128 p.

Murray John, 2007. The Minnows of Triton. Policing, Politics, Crime and Corruption in the South Pacific Islands, Canberra, 297 p.

Panoff Michel, Tahiti métisse, Paris, Denoël, 1989, 296 p.

PoIrine Bernard, 2011. Tahiti: une économie sous serre, Paris, L'Harmattan, $196 \mathrm{p}$.

Poirson Brune et Nicolas Miailhe, 2014 (février-mars). Vers un Big Bang technologique ? CCE International 572, pp. 18-26.

Rancière Jacques, 2004. Aux bords du politique, Paris, Folio, 272 p. 
Regnault Jean-Marc (éd.), 2003. François Mitterrand et les territoires français du Pacifique (1981-1988) : mutations, drames et recompositions; enjeux internationaux et franco-français, Paris, Les Indes savantes, 583 p.

Regnault Jean-Marc, 2013. La France, l'ONU et les décolonisations tardives. L'exemple des terres françaises d'Océanie, Aix, PUAM, 250 p.

Richer de Forges Bertrand et Michel Pascal, 2008. La Nouvelle-Calédonie: un "point chaud » de la biodiversité mondiale gravement menacé par l'exploitation minière, Journal de la Société des Océanistes 126-127, pp. 95-112 (http://jso.revues.org/4052).

RIFKIN Jeremy, 2011. La troisième révolution industrielle. Comment le pouvoir latéral va transformer l'énergie, l'économie et le monde, Paris, Éd. Les Liens qui libèrent, 380 p.

Rigo Bernard, 2007. Le pouvoir politique et le sacré en Polynésie, in Chatti Mounira et al., Pouvoir (s) et politique (s) en Océanie, Paris, L'Harmattan, pp. 297-322.

Robineau Claude, 1978. Réciprocité, redistribution et prestige chez les Polynésiens des îles de la Société, Journal de la Société des Océanistes 61-34, pp. 161-168. (http://www. persee.fr/web/revues/home/prescript/article/ jso_0300-953x_1978_num_34_61_2980)

Robineau Claude, 1985. Tradition et modernité aux îles de la Société, Paris, ORSTOM, 790 p.

RoYer Pierre, 2012. Géopolitique des mers et des océans. Qui tient la mer tient le monde, Paris, PUF, 208 p.

Saura Bruno, 2008. Tahiti Ma'ohi. Culture, identité, religion et nationalisme en Polynésie française, Papeete, Au Vent des îles, 529 p.

SeILer Daniel-Louis, 2006. L'échange, le politique et le marché : réflexions maritimes, Revue internationale de politique comparée 4, 13, pp. 589-616 (http://www.cairn.info/ revue-internationale-de-politique-comparee2006-4-page-589.htm).

SÉnat, 2013 (17/01). La France dans le Pacifique : quelle vision pour le XXIe siècle?, rapport $\mathrm{n}^{\circ} 293,144$ p. (http://www.senat.fr/noticerapport/2012/r12-293-notice.html).

Serra-Mallol Christophe, 2010. Changement social et traditions alimentaires. Socio-anthro- pologie de l'alimentation à Tahiti, Papeete, Au Vent des îles, $543 \mathrm{p}$.

Stiglitz Joseph, 2010 (2008). Le triomphe de la cupidité, Paris, Éd. Les Liens qui libèrent, $473 \mathrm{p}$.

Smith Andy et Claude Sorbets (éds), 2003. Le leadership politique et le territoire. Les cadres d'analyse en débat, Rennes, Presses Universitaires de Rennes, 293 p.

TAGLIONI François, 2007. Les petits espaces insulaires au cœur des revendications frontalières maritimes dans le monde, L'Espace politique 1 (http://espacepolitique.revues.org/732).

TJibaou Jean-Marie, 1985. Entretien avec JeanMarie Tjibaou, Les Temps modernes 464: Nouvelle-Calédonie, pour l'indépendance, pp. 1587-1601.

TRICHet Jean et Isabelle LeBLIC (éds), 2008. Journal de la Société des Océanistes 126-127: L'environnement dans le Pacifique, 166 p. (http://jso.revues.org/1804).

Weber Max, 2004 (1921). Economie et société, 2 volumes, Paris, Plon/Agora.

White Gregory, 2011. Climate Change and Migration: Security and Borders in Warming World, Oxford, Oxford University Press.

Wittersheim Éric, 2014. Noir c'est noir: l' "Africanisation " du Pacifique en question, in L. Dousset et al. (éds), Les sciences humaines et sociales dans le Pacifique Sud. Terrains, questions et méthodes, Marseille, Pacific Credo Publications, Cahiers du Credo, pp. 377-400.

—, 2006. Après l'indépendance. Le Vanuatu, une démocratie dans le Pacifique, Montreuil, Aux lieux d'être, $190 \mathrm{p}$.

Wolton Dominique et al. (éds), 2004. Hermès 40 : Francophonie et mondialisation, $412 \mathrm{p}$.

WorLiczeK Elisabeth, 2013. La vision de l'espace littoral sur l'île Wallis et l'atoll Rangiroa dans le contexte du changement climatique. Une analyse anthropologique de la perception des populations locales, thèse d'anthropologie culturelle et sociale, sous la dir. de M. Allenbach et H. Mückler, Université de la Nouvelle-Calédonie/Université de Vienne (http:// www.theses.fr/2013NCAL0049). 